\title{
Genetic divergence between two sympatric species of the Lutzomyia longipalpis complex in the paralytic gene, a locus associated with insecticide resistance and lovesong production
}

\author{
RMMA Lins, NA Souza', AA Peixoto/ ${ }^{+}$ \\ Laboratório de Biologia Molecular de Insetos ' ${ }^{1}$ aboratório de Transmissores de Leishmanioses, Instituto Oswaldo Cruz-Fiocruz, \\ Av. Brasil 4365, 21045-900 Rio de Janeiro, RJ, Brasil
}

The sandfly Lutzomyia longipalpis s.l. is the main vector of American Visceral Leishmaniasis. L. longipalpis s.l. is a species complex but until recently the existence of cryptic sibling species among Brazilian populations was a controversial issue. A fragment of paralytic (para), a voltage dependent sodium channel gene associated with insecticide resistance and courtship song production in Drosophila, was isolated and used as a molecular marker to study the divergence between two sympatric siblings of the L. longipalpis complex from Sobral, Brazil. The results revealed para as the first single locus DNA marker presenting fixed differences between the two species in this locality. In addition, two low frequency amino-acid changes in an otherwise very conserved region of the channel were observed, raising the possibility that it might be associated with incipient resistance in this vector. To the best of our knowledge, the present study represents the first population genetics analysis of insecticide resistance genes in this important leishmaniasis vector.

Key words: Lutzomyia longipalpis - Drosophila - insecticide resistance - para - Leishmaniasis - sandflies - sympatric species

The sandfly Lutzomyia longipalpis s.l. (Lutz \& Neiva 1912) (Diptera: Psychodidae: Phlebotominae) is the main vector of Leishmania chagasi (= Leishmania infantum), the causative agent of American Visceral Leishmaniasis. Because of its importance as a vector, several studies have been carried out to verify the taxonomic status of $L$. longipalpis and there is now a large amount of data indicating that this sandfly is a species complex (reviewed in Bauzer et al. 2007, Maingon et al. 2008). Although the current evidence clearly indicates the existence of sibling species among Brazilian populations, the issue was a controversial one until recently (Bauzer et al. 2007, Maingon et al. 2008).

The best evidence for the existence of sibling species in Brazil can be found in Sobral (state of Ceará). In this locality males of two sympatric species can be differentiated by the presence of one (1S) or two (2S) abdominal spots (Ward et al. 1988, Souza et al. 2008), produce different types of pheromones and copulatory courtship songs (Souza et al. 2004, Hamilton et al. 2005) and show a significant genetic divergence with different molecular markers (Bauzer et al. 2002a, Maingon et al. 2003, Bottecchia et al. 2004).

Genes controlling the production of acoustic signals during courtship are interesting molecular markers to study the speciation process since these "lovesongs" are

Financial support: Howard Hughes Medical Institute, CNPq, Fiocruz (PAPES IV)

+Corresponding author: apeixoto@fiocruz.br

Received 27 May 2008

Accepted 29 September 2008 an important aspect of sexual behaviour and reproductive isolation in many insect species. A number of genes controlling the courtship song in Drosophila melanogaster have been identified (Gleason 2005) and two of these, period and cacophony, have already been used in studies of the L. longipalpis complex in Brazil (Bauzer et al. 2002a,b, Bottecchia et al. 2004).

One of the genes controlling the lovesong in $D$. melanogaster is paralytic (para) (Peixoto \& Hall 1998). Interestingly, mutations in para orthologues are also associated with insecticide resistance in a number of organisms (Hemingway \& Ranson 2000, Hemingway et al. 2004). This gene codes for the $\alpha$ subunit of a voltage-dependent sodium channel (Loughney et al. 1989, Littleton \& Ganetzky 2000) and its homologues in other insects are the site of a number of mutations associated with resistance to DDT and pyrethroids, including the well known $k d r$ mutation (Milani 1956, Loughney et al. 1989, Pittendrigh et al. 1997).

Insecticide resistance in vectors of tropical diseases is a major public health problem. Nevertheless, in many vector species such as Neotropical sandflies the occurrence of insecticide resistance is still poorly studied. The use of resistance genes as molecular markers offers an attractive approach to investigate the rise and spread of insecticide resistance mutations in populations of vector species.

Interestingly some authors speculate whether insecticide resistance is a consequence of insecticide introduction or if these mutations arose before it (Hartley et al. 2006, ffrench-Constant 2007). It has been shown that mutations conferring resistance to Malathion in blowflies of the genus Lucilia were already present before the introduction of this insecticide (Hartley et al. 2006). This implies that species carrying such alleles are preadapted to insecticide exposition. 
The occurrence of insecticide resistance in L. longipalpis has not yet been reported. There are only a handful of studies on this subject in sandflies (e.g. Rahman et al. 1982, Mukhopadhyay et al. 1987, 1996, Tetreault et al. 2001, Surendran et al. 2005, Coutinho-Abreu et al. 2007) and further research is needed, particularly in Neotropical species. Therefore, the isolation of $L$. longipalpis molecular markers homologous to genes controlling insecticide resistance in other insects might help to elucidate this question.

We have used a degenerate primer PCR approach to clone a L. longipalpis fragment homologous to the para gene of Drosophila. The primers were designed to amplify the region encoding the IIS6 transmembrane segment of the channel known to contain insecticide resistant mutations in other insects. A population genetic analysis was also carried out using the two sympatric species of the locality of Sobral.

A sample of L. longipalpis from a laboratory colony originated from sandflies collected at Lapinha cave (state of Minas Gerais, Brazil) was used to isolate the initial fragment. The samples from Sobral were the same analysed by Bauzer et al. (2002a) and Bottecchia et al. (2004).

For the initial fragment of the para gene, mRNA was isolated with QuickPrep ${ }^{\mathrm{TM}}$ Micro mRNA purification kit (Amersham Biosciences) and cDNA was synthesized using the First-Strand cDNA synthesis kit (Amersham Biosciences). Initially, touchdown PCR was performed in $40 \mu \mathrm{L}$ using Promega reagents with various cycling conditions and the degenerate primers: 5paraIIdegC $\left(5^{\prime}\right.$ - TGGAAYTTYACNGAYTT - $\left.3^{\prime}\right)$ and 3paraIIdegB $\left(5^{\prime}\right.$ - TTRTTNGTRTCRTTRTC - $\left.3^{\prime}\right)$. Degenerate primers were designed based on conserved regions identified by alignment of the protein sequences encoded by para orthologues in different insect species. Based on the initial fragment generated, specific primers were designed.

For the population genetics analyses of the two Sobral siblings, genomic DNA of individual flies were isolated according to Jowett (1998) and the PCR Master Mix (Promega) was used to perform reactions according to the following cycling conditions: $95^{\circ} \mathrm{C}$ for $3 \mathrm{~min} ; 35$ cycles at $95^{\circ} \mathrm{C}$ for $30 \mathrm{~s}, 60^{\circ} \mathrm{C}$ for $30 \mathrm{~s}$ and $72^{\circ} \mathrm{C}$ for $30 \mathrm{~s}$. Specific primers used were: 51 para 2 (5' - ACGGACTTCATGCATTCATTC - 3') and 3llpara1 (5' - TGGTGCTGATAAACTTGACG - 3').
PCR products were purified using the Wizard SV Gel and PCR Clean-up System (Promega) and cloned using the pMOSBlue Blunt Ended Cloning Kit (Amersham Biosciences). Plasmidial DNA was isolated using Flexiprep Kit (Amersham Biosciences) or using 96 wells microplates and the alcaline lysis method followed by filtration in Millipore Multiscreen filter plates. DNA sequencing was carried out in ABI377 and ABI 3730 Sequencers using the Big Dye 3.1 Kit (Applied Biosystems). Homology to the Drosophila para gene was confirmed by comparison with databanks using BlastX (http://www.ncbi.nlm.nih.gov).

Fragments of the para gene of $L$. longipalpis from Sobral $1 \mathrm{~S}$ and $2 \mathrm{~S}$ were initially processed using GCG program (Wisconsin Package Version 9.1, Genetic Computer Group, Madison, Wisconsin, USA) before the population genetics analyses. A minimum of three and maximum of eight sequences per individual were aligned to obtain one or, when possible, two consensus sequences corresponding to the two alleles, named as A and B. Polymorphism and population structure analyses were carried out using both DnaSP 4.10.4 (Rozas et al. 2003) and Proseq 2.9 (Filatov \& Charlesworth 1999) programs. Phylogenetic analysis was performed using Mega 3.1 (Kumar et al. 2004). Sequences were submitted to the GenBank (accession numbers DQ914434; EU746318-EU746365).

Fig. 1 shows the amino acid sequence encoded by the initial fragment obtained from $L$. longipalpis aligned with the respective region of the para protein of D. melanogaster. This region is highly conserved with only one amino acid substitution $(\mathrm{Y} \rightarrow \mathrm{L})$ in $L$. longipalpis. The intron position is also conserved.

We have generated 27 consensus sequences of Sobral 1S and 21 sequences of Sobral 2S, where 376 sites were analyzed and 23 of them (6.1\%) were variable. Fig. 2 shows the alignment of polymorphic sites observed in the different haplotypes generated. Most of the variation found is within the intron which also shows a few insertion deletion events. We also have found one synonymous (position 19) and two non-synonymous substitutions (position 114, $\mathrm{N} \rightarrow \mathrm{K}$, Sob2S17B and position 369, $\mathrm{N} \rightarrow \mathrm{K}$, Sob2S14A). The positions of these two non-synonymous substitutions are underlined in the alignment shown in Fig. 1. Compared to the other two lovesong
D. melanogaster
L. longipalpis

D. melanogaster

L. longipalpis

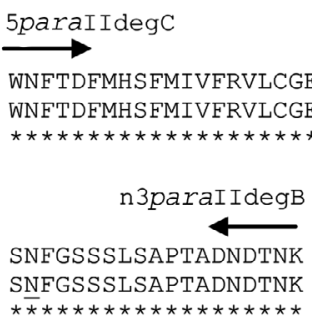

$* \bar{*} * * * * * * * * * * * * * * * * *$

Fig. 1: alignment of the amino acid sequence encoded by para gene fragment from Lutzomyia longipalpis and Drosophila melanogaster. Arrows point to the approximate location of the primers used. The position of the intron found in the genomic sequences of D. melanogaster and L. longipalpis is indicated by an inverted triangle. The position where most mutations associated with insecticide resistance to DDT and pyrethroids in D. melanogaster and other insects occur is highlighted. Asterisks below the alignment represent positions with identical amino acids between the two species. The position of the non-synonymous substitutions found in Sobral 2S are underlined (see text for details). 
genes analysed in Sobral (Bauzer et al. 2002a, Bottecchia et al. 2004), the level of polymorphism in para is low ( $\pi=0.0037$ and $\theta=0.0091$ for Sobral 1S and $\pi=0.0045$ and $\theta=0.0075$ for Sobral $2 S$ ). $F_{S T}$ analysis showed a very high and significant differentiation between Sobral $1 \mathrm{~S}$ and Sobral $2 \mathrm{~S}\left(F_{S T}=0.7852 ; \mathrm{Nm}=0.07, \mathrm{p}<0.0001\right)$. In addition, three fixed differences were observed between the two siblings.

Fig. 3 shows a minimum evolution tree of the sequences of the two sympatric siblings. This tree shows a clear separation between the sequences of the two species with a high bootstrap value of $91 \%$. The tree obtained shows a similar topology to that using the period gene (Bauzer et al. 2002a).

1111112222222222222233

11678991123456666777827

94591165762210248368460

sob2s11A TTCTGGCTCTGTTCATCTATCTT

sob2s13A ...................

sob2s14A ....T.....TC.... CA

sob2s15A ....................

sob2s16A ...................

sob2s16B ....T.....TC..... .

sob2s17A …T. ..............

sob2s17B .G...T.....T.G...TC.

sob2s18A ..................

sob2s18B .................. .

sob2s1A …T...............

sob2s4A ................TC.

sob2s 4 B ..................

sob2s5A .................. .

sob2s6A …T........... TC.

sob2s7A ……............

sob2s7B ……........

sob2s8A …T............

sob2s8B …T..... . . . TC.

sob2s9A …T.....TCG.....

sob2s9B …T..............

SOb1s10A C.TG.T.... СATC......

sob1s10B C.CG..T....CATC......

soblsilA C..G.......АТCT..... .

sob1s12A C..G..TC...CATC.......

Sob1s12B C..G..TT...CATC...... .

sobls13A C..G........ AATC........

sob1s14A C..G..T...CATC.......

sobls15A C..G..T...CATC......

soblsi5B C..G.T...ACATC..G...C.

SOb1s16A C..G..T....CATC.......

sob1s16B C..G.T...ACATC..G...C.

SOb1s17A C..G.T..A.CATC.G.....

sobls17B C..G.AT...CATC.......

SOb1s18A C..GA.T...CATC......

sob1s18B C..G.TT...CATC...... .

sob1s1A C..G.T.T..CATC..T.T.C.

sob1s1B C..G.T....CATC..A.G.C.

sob1s2A C......... CATC......

sobls2B C..GA.T...CATC......

sob1s4A C........... AATC.......

sob1s5A C....T....CATC.......

sobls5B C..G..T...CATC...... .

SOb1s6A C..G.T...CATC..A.G.C.

sobls6B C..G.T....СATC......

sob1s8A C..G.T..T.CATCT.....

sob1s8B C...........АTCT.....

sob1s9A C....T....СATC......

Fig. 2: variable sites alignment of para gene fragment consensus sequences of Lutzomyia longipalpis from Sobral. Sites occurring in the exon are shown in bold. Dots indicate the same nucleotide as in the first aligned sequence: sob1s: Sobral one spot; sob2s: Sobral two spots. The three fixed differences between the two siblings are highlighted.
Although insecticide resistance has not yet been demonstrated in L. longipalpis there are some indications of its occurrence in this species (Falcão et al. 1988, Mazzarri et al. 1997). The fragment we isolated may provide a useful molecular marker for the screening of putative insecticide resistance mutations in populations of this vector. In fact, although the non-synonymous substitutions observed in Sobral 2S were not associated with

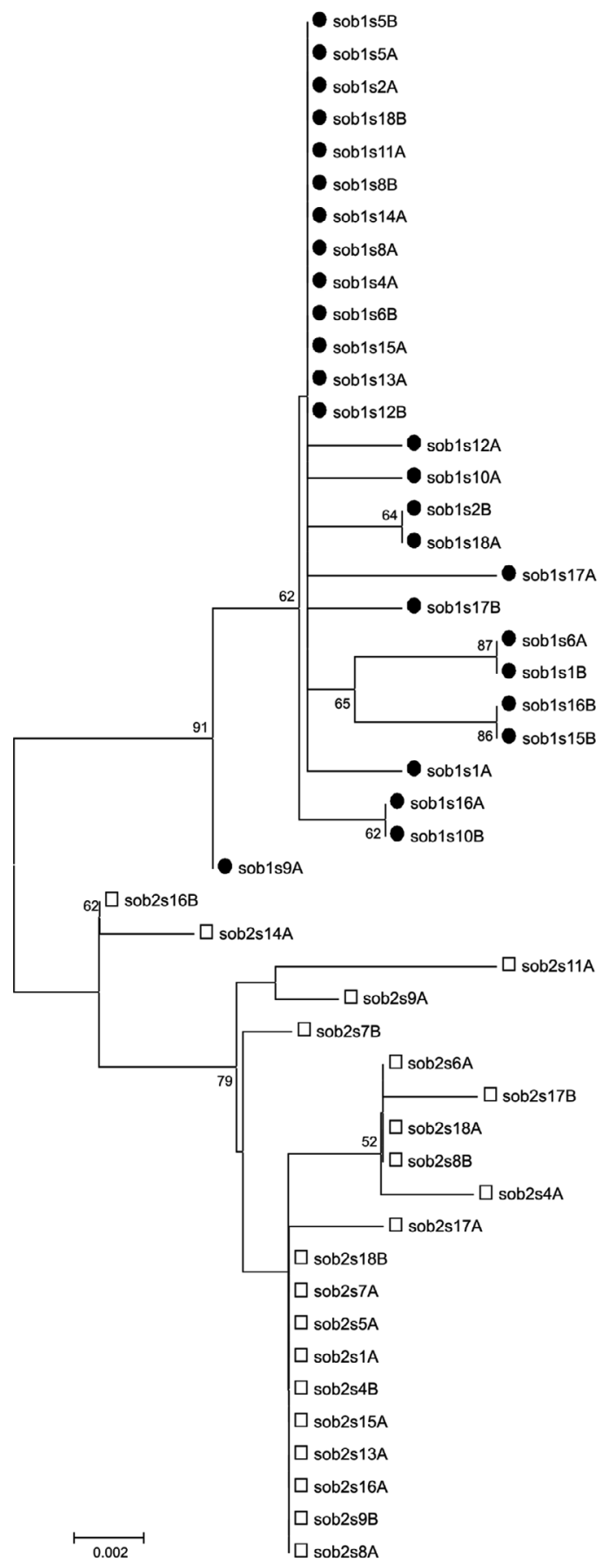

Fig. 3: minimum evolution tree constructed using p-distance and the para sequences of Sobral 1S (black circles) and $2 \mathrm{~S}$ (white squares). Bootstrap values were obtained with 1000 permutations. Only values above $50 \%$ are shown. 
insecticide resistance in other species, these amino-acid changes occur in an otherwise very conserved region of the channel, raising the possibility that it might be associated with incipient resistance in this vector. Therefore, it might be interesting to screen other populations, especially those under current treatment with pyrethroids, for the presence of these mutations.

As mentioned above, L. longipalpis is a species complex (Bauzer et al. 2007, Maingon et al. 2008) and males of different sibling species produced very distinct copulatory courtship songs (Souza et al. 2004). In addition to its role in insecticide resistance, para is also involved in the control of the courtship song in Drosophila (Peixoto \& Hall 1998). Therefore para is a very good candidate as an additional molecular marker for further studies in this species complex. Although fixed differences in isoenzyme loci have been reported (e.g., Lanzaro et al. 1993, Lampo et al. 1999) and microsatellites have been used successfully in the genetic identification of L. longipalpis siblings (Maingon et al. 2003), para is the first single locus DNA marker presenting fixed differences between the two sympatric species from Sobral. This might allow the development of a field friendly agarose assay to differentiate the siblings. Therefore this locus might be especially useful as a tool to species identification of females in field studies aimed at comparing the infection rates and vectorial capacity between these two sympatric sibling species of the $L$. longipalpis complex.

\section{REFERENCES}

Bauzer LGSR, Gesto JSM, Souza NA, Ward RD, Hamilton JGC, Kyriacou CP, Peixoto AA 2002a. Molecular divergence in the period gene between two putative sympatric species of the Lutzomyia longipalpis complex. Mol Biol Evol 19: 1624-1627.

Bauzer LGSR, Souza NA, Maingon RDC, Peixoto AA 2007. Lutzomyia longipalpis in Brazil: a complex or a single species? A minireview. Mem Inst Oswaldo Cruz 102: 1-12.

Bauzer LGSR, Souza NA, Ward RD, Kyriacou CP, Peixoto AA 2002b. The period gene and genetic differentiation between three Brazilian populations of Lutzomyia longipalpis. Insect Mol Biol 11: 315-323.

Bottecchia M, Oliveira SG, Bauzer LG, Souza NA, Ward RD, Garner KJ, Kyriacou CP, Peixoto AA 2004. Genetic divergence in the cacophony IVS6 intron among five Brazilian populations of Lutzomyia longipalpis. J Mol Evol 58: 754-761.

Coutinho-Abreu IV, Balbino VQ, Valenzuela JG, Sonoda IV, Ramalho-Ortigão JM 2007. Structural characterization of acetylcholinesterase 1 from the sand fly Lutzomyia longipalpis (Diptera: Psychodidae). J Med Entomol 44: 639-650.

Falcão AL, Pinto CT, Ferreira Gontijo CM 1988. Susceptibility of Lutzomyia longipalpis to deltamethrin. Mem Inst Oswaldo Cruz 83: 395-396.

ffrench-Constant RH 2007. Which came first: insecticides or resistance? Trends Genet 23: 1-4.

Filatov DA, Charlesworth D 1999. DNA polymorphism, haplotype structure and balancing selection in the Leavenworthia PgiC locus. Genetics 153: 1423-1434.

Gleason JM 2005. Mutations and natural genetic variation in the courtship song of Drosophila. Behav Genet 35: 265-277.
Hamilton JG, Maingon RD, Alexander B, Ward RD, Brazil RP 2005. Analysis of the sex pheromone extract of individual male Lutzomyia longipalpis sandflies from six regions in Brazil. Med Vet Entomol 19: 480-488.

Hartley CJ, Newcomb RD, Russell RJ, Yong CG, Stevens JR, Yeates DK, La Salle J, Oakeshott JG 2006. Amplification of DNA from preserved specimens shows blowflies were preadapted for the rapid evolution of insecticide resistance. Proc Natl Acad Sci USA 103: 8757-8762.

Hemingway J, Hawkes NJ, McCarroll L, Ranson H 2004. The molecular basis of insecticide resistance in mosquitoes. Insect Biochem Mol Biol 34: 653-665.

Hemingway J, Ranson H 2000. Insecticide resistance in insect vectors of human disease. Annu Rev Entomol 45: 371-391.

Jowett T 1998. Preparation of nucleic acids. In DB Roberts (ed.), Drosophila: A practical approach, IRL Press, Oxford, p. 347-371.

Kumar S, Tamura K, Nei M 2004. MEGA3: Integrated software for Molecular Evolutionary Genetics Analysis and sequence alignment. Brief Bioinform 5: 150-163.

Lampo M, Torgerson D, Márquez LM, Rinaldi M, García CZ, Arab A 1999. Occurrence of sibling species of Lutzomyia longipalpis (Diptera: Psychodidae) in Venezuela: first evidence from reproductively isolated sympatric populations. Am J Trop Med Hyg 61: 1004-1009.

Lanzaro GC, Ostrovska K, Herrero MV, Lawyer PG, Warburg A 1993. Lutzomyia longipalpis is a species complex: genetic divergence and interspecific hybrid sterility among three populations. Am J Trop Med Hyg 48: 839-847.

Littleton JT, Ganetzky B 2000. Ion channels and synaptic organization: analysis of the Drosophila genome. Neuron 26: 35-43.

Loughney K, Kreber R, Ganetzky B 1989. Molecular analysis of the para locus, a sodium-channel gene in Drosophila. Cell 58: 1143-1154.

Maingon RD, Ward RD, Hamilton JG, Bauzer LG, Peixoto AA 2008. The Lutzomyia longipalpis species complex: does population sub-structure matter to Leishmania transmission? Trends Parasitol 24: 12-17.

Maingon RD, Ward RD, Hamilton JG, Noyes HA, Souza N, Kemp SJ, Watts PC 2003. Genetic identification of two sibling species of Lutzomyia longipalpis (Diptera: Psychodidae) that produce distinct male sex pheromones in Sobral, Ceará State, Brazil. Mol Ecol 12: 1879-1894.

Mazzarri MB, Feliciangeli MD, Maroli M, Hernandez A, Bravo A 1997. Susceptibility of Lutzomyia longipalpis (Diptera: Psychodidae) to selected insecticides in an endemic focus of visceral leishmaniasis in Venezuela. J Am Mosq Control Assoc 13: 335-341.

Milani R 1956. Mendelian behavior of resistance to the knock-down action of DDT and correlation between knock-down and mortality in Musca domestica L. Rend Ist Sup Sanit 19: 1107-1143.

Mukhopadhyay AK, Chakravarty AK, Kureel VR, Shivraj 1987. Resurgence of Phlebotomus argentipes \& Ph. papatasi in parts of Bihar (India) after DDT spraying. Ind J Med Res 85: 158-160.

Mukhopadhyay AK, Hati AK, Chakraborty S, Saxena NB 1996. Effect of DDT on Phlebotomus sandflies in Kala-Azar endemic foci in West Bengal. $J$ Commun Dis 28: 171-175.

Peixoto AA, Hall JC 1998. Analysis of temperature-sensitive mutants reveals new genes involved in the courtship song of Drosophila. Genetics 148: 827-838.

Pittendrigh B, Reenan R, ffrench-Constant RH, Ganetzky B 1997. 
Point mutations in the Drosophila sodium channel gene para associated with resistance to DDT and pyrethroid insecticides. Mol Gen Genet 256: 602-610.

Rahman SJ, Wattal BL, Mathur KK, Joshi GC, Kumar K 1982. Susceptibility of laboratory reared strain of Phlebotomus papatasi (Scopoli) to organochlorine insecticides. J Commun Dis 14: 122-124.

Rozas J, Sánchez-DelBarrio JC, Messeguer X, Rozas R 2003. DnaSP, DNA polymorphism analyses by the coalescent and other methods. Bioinformatics 19: 2496-2497.

Souza NA, Andrade-Coelho CA, Vigoder FM, Ward RD, Peixoto AA 2008. Reproductive isolation between sympatric and allopatric Brazilian populations of Lutzomyia longipalpis s.l. (Diptera: Psychodidae). Mem Inst Oswaldo Cruz 103: 216-219.

Souza NA, Vigoder FM, Araki AS, Ward RD, Kyriacou CP, Peixoto
AA 2004. Analysis of the copulatory courtship songs of Lutzomyia longipalpis in six populations from Brazil. $J$ Med Entomol 41: 906-913.

Surendran SN, Karunaratne SH, Adams Z, Hemingway J, Hawkes NJ 2005. Molecular and biochemical characterization of a sand fly population from Sri Lanka: evidence for insecticide resistance due to altered esterases and insensitive acetylcholinesterase. Bull Entomol Res 95: 371-380.

Tetreault GE, Zayed AE, Hanafi HA, Beavers GM, Zeichner BC 2001. Susceptibility of sand flies to selected insecticides in North Africa and the Middle East. J Am Mosq Control Assoc 17: 23-27.

Ward RD, Phillips A, Burnet B, Marcondes CB 1988. The Lutzomyia longipalpis complex: reproduction and distribution. In MW Service, Biosystematics of Haematophagus Insects, Oxford University Press, Oxford, pp. 258-269. 